\title{
Fast random wave generation in numerical tanks
}

\section{Aggelos S. Dimakopoulos ${ }^{1}$, Tristan de Lataillade ${ }^{1}$ and Chris E. Kees ${ }^{2}$}

${ }^{1}$ HR Wallingford, Howbery Park, Wallingford, Oxfordshire OX10 8BA, UK

2 ERDC, 3909 Halls Ferry Rd, Vicksburg, MS 39180, United States

Corresponding author: Aggelos Dimakopoulos, a.dimakopoulos@hrwallingford.com

Proceedings of the Institution of Civil Engineers - Engineering and Computational Mechanics, Volume 172, Issue 1, PP 1-11 (March 2019)

\section{Abstract}

Generating and absorbing random waves in numerical models is a challenging problem, in particular when meaningful wave statistics should be generated to meet design sea state requirements. The methodology presented herein allows for the generation of random wave fields (free surface elevation and velocities) to be reconstructed in time and in space by using window processing from a reference time series. It is demonstrated that the methodology is efficient in reproducing long non-repeating wave sequences by using only $\mathrm{O}(101)-\mathrm{O}(102)$ wave components, rather than $\mathrm{O}(103)-\mathrm{O}(104)$ required by a direct reconstruction from a single spectrum. This reduces the computational times required for the development of wave-train time series elements by 40 times. Errors in instantaneous surface elevation and particle velocity between windowed and non-windowed reconstruction techniques were less than $0.4 \%$ and $0.2 \%$ respectively in the cases considered. The technique was combined with the relaxation zone method typically used in numerical wave tanks for generating waves. The simulations were performed using Proteus, a rapidly developing CFD - FEM toolkit for modelling fluid structure interaction cases. The use of windowed reconstruction reduced the overall computational time associated with the simulation of waves in a numerical wave tank by $\sim 40 \%$ and $\sim 70 \%$ for serial and parallel execution. Results of the study show windowed reconstruction to be suitable for the representation of long-duration wave trains. Wave height and peak period are conserved within $2 \%$ and $1 \%$ respectively.

\section{Keywords}

coastal engineering; mathematical modelling; fluid mechanics 


\section{Introduction}

Recently, numerical wave tanks in Computational Fluid Dynamics (CFD) models have been increasingly employed to assist the design optimisation of coastal structures, along with physical modelling data (Richardson et al. 2013, Cuomo et al. 2013). Typical configurations for numerical wave tanks can be found in Jacobsen et al (2012), Higuera et al (2013), Chen et al. (2015), Schmitt and Elsaesser, B (2015) and Dimakopoulos (2016) using different techniques for generating and absorbing waves such as moving paddles, radiation boundary conditions, and relaxation zones.

In CFD models using the relaxation zone method, free-surface elevation and wave velocities are typically calculated within a subdomain adjacent to the offshore boundary. As a result of the sizable generation domain there is a significant computational cost associated with the generation of input surface elevations and wave velocities to be applied in both time and space. In order to reduce these costs, Dimakopoulos et al (2016) improved and further tested the relaxation zone technique developed by Jacobsen et al (2012). Improvements were made by replacing the built-in trigonometric functions with faster approximations based on Taylor theory. Numerical benchmarks were performed using 500 wave components for signal reconstruction and showed that simple optimisation techniques could reduce the computational cost by one order of magnitude ( $\sim 90 \%)$. In terms of total simulation time Dimakopoulos et al (2016) proved that employing the optimised technique may reduce the total simulation time by $\sim 45 \%$, for a 45 m numerical wave tank discretised with 240000 cells. Despite the reductions achieved, the relative computational burden of wave generation remains relatively high, as for example increasing the frequency components from 50 to 500 yields a $40 \%$ increase in the total simulation time (Dimakopoulos et al 2016). Generating non-repeating wave series of e.g. 1000 waves (typical storm duration)will require more than $O\left(10^{3}\right)$ wave components to be calculated for every time step, and it is evident that this will significantly increase the computational cost required. This number will increase even further when considering nonlinear corrections to the random wave series, in order to calculate $2^{\text {nd }}$ order nonlinear wave-wave interactions (Tucker 1995; Schaffer 1996; Dalzell 1999).

This work proposed a methodology that will enable generation of non-repeating wave sequences using significantly fewer frequency components $\left(<<10^{3}\right)$ for reconstructing wave field parameters typically used as inputs for CFD simulations. This is achieved by pre-processing the free surface elevation time series before the start of the simulation using windowing techniques (Harris 1978) that will allow an accurate spectral decomposition using a significantly reduced number of components. In this paper it is assumed that spectra are discretised using a constant frequency step. The technique could be generalised to include other discretisation methods (e.g. see Miles and Funke 1988).

The methodology is coupled with the computational toolkit Proteus (http://proteustoolkit.org/) to demonstrate its use within the frame of a CFD model. Proteus is a rapidly developing, open-source finite-element based toolkit that is able to model wave propagation and absorption using a coupled level-set / volume of fluid method (Kees et al. 2008, 2009 and 2011). The relaxation zone method is used for wave generation / absorption, that has been widely employed for similar purposes in various numerical tools such as CFD models (Mayer et al 1998, Jacobsen et al 2012) and depth-integrated models, e.g. BOUSS2D (Nwogu and Dirbilek 2001) or FUNWAVE (Shi et al. 2016). It is nevertheless envisaged that the methodology will be suitable for generating time series for boundary conditions in similar depth-integrated and other types of models, after appropriate modifications (e.g. use of depth integrated velocity rather than particle velocity). As Proteus is currently being developed for simulating fluid-structure interaction problems, an additional objective is to establish a robust and efficient technique to generate and absorb random waves for this 
particular model. Relevant results recently presented in Dimakopoulos et al. (2017a; 2017b) concerning wave and current interaction with moving structures (e.g. sliding breakwaters, flow around oscillating pipelines, floating objects) reveal the potential for Proteus to be used for assisting the design of marine and coastal structures.

This paper is structured as follows: In Section 2, the methodology of processing and reconstructing a wave series to reduce the computational cost associated with the reconstruction is presented in addition to coupling of the methodology with the relaxation zone method. In Section 3 the overall benefits of optimisation are quantified using simple benchmark cases. In Section 4, validation results are presented in terms of random wave propagation and improvement of the overall simulation times are demonstrated. Section 5 discusses conclusions, potential issues and future work regarding the methodology and the numerical modelling approach.

\section{Methodology}

\subsection{Reconstruction of components with window method}

According to linear theory, free surface elevation and velocity components for a plane random wave field can be calculated using discrete linear reconstruction of components, as shown in equation 1 to 3 .

$\eta(\vec{x}, t)=\sum_{i=1}^{N} a_{i} \cos \left(\vec{k}_{i} \cdot \vec{x}-\omega_{i} t+\phi_{i}\right)$

$U_{h}(\vec{x}, z, t)=\sum_{i=1}^{N} u_{h(i)} \cos \left(\vec{k}_{i} \cdot \vec{x}-\vec{\omega}_{i} t+\phi_{i}\right) \cosh \left(k_{i}(d+z)\right)$

$U_{v}(\vec{x}, z, t)=\sum_{i=1}^{N} u_{v(i)} \sin \left(\vec{k}_{i} \cdot \vec{x}-\vec{\omega}_{i} t+\phi_{i}\right) \sinh \left(k_{i}(d+z)\right)$

where $\eta$ is the free-surface elevation, $\vec{x}$ the position vector at the wave propagation plane, $z$ is the vertical coordinate (vertical axis aligned with gravity), $t$ the time variable, $U_{h}$ and $U_{v}$ is the horizontal and vertical velocity, respectively, and $a_{i}, \vec{k}_{i}, \omega_{i}, \phi_{i}, u_{h(i)}$ and $u_{v(i)}$ are the wave amplitude, wave number vector, angular frequency, phase and horizontal and vertical velocity amplitude, respectively, for the $i$-th wave component and $N$ is the number of frequency components. The wave amplitude for each component is calculated from the spectral distribution according to equation 4.

$a_{i}=\sqrt{2 S_{i} \Delta f_{i}}$

where $S_{i}$ and $\Delta f_{i}$ are the discrete spectral energy distribution and frequency interval for the $i$-th linear component. The discrete spectral energy distribution typically follows established distributions for random sea waves e.g. JONSWAP or Pierson-Moskowitch. In this work, the JONSWAP spectrum is used.

In order to ensure a non-repeating random wave series, the discretisation step of the spectrum in the frequency domain $(\Delta f)$ must be set according to equations 5 and 6 (Dean and Dalrymple, 1994). 
$\Delta f=\frac{f_{p} b_{F}-\frac{f_{p}}{b_{F}}}{N}$

$T_{\max }=\frac{1}{\Delta f}$

where $b_{F}$ is the spectral band factor so that the frequency components belong to the interval $\left[\frac{f_{p}}{b_{F}}, f_{p} b_{F}\right]$, where $f_{p}$ is the peak frequency of the random wave spectrum and $T_{\text {max }}$ is the maximum duration of a nonrepeating wave sequence. Since $b_{F}$ varies within a relatively limited range (typically $2 \sim 3$ ), the length of the non-repeating sequence is rather controlled by the total number of the frequency components and in particular, finer discretisation of the spectrum (using a larger number of wave components) is required to achieve larger non-repeating wave sequences. For example, a non-repeating sequence for 1000 waves, would approximately require $\sim 1500$ and $\sim 2500$ frequency components, for $b_{F}$ equal to 2 and 3 , respectively.

In order to reduce the computational cost, rather than directly processing the time series of free surface elevation using Equations 1 and 2 at each timestep, we introduce a novel reconstruction methodology based on signal processing using windows, also known as the smoothed periodogram method or Welch's method (Welch 1967). Window methods are typically used for processing signals and calculating smoothed spectral distributions. Herein it is used for pre-processing a free surface elevation time series and generating the wave field using a reduced number of frequencies.

For initialising the process, a non-repeating time series is generated for the free surface elevation using Equation 1 with an appropriate number of frequencies components, according to Equation 4 . The direct reconstruction is performed only once before the initialisation of the simulation and only for the free surface elevation. Once generated, the time series should be sub-divided into separate windows, each with a span ideally much shorter than the total duration.. Each window is then decomposed into a discrete spectrum by using Discrete Fourier Transform (DFT) after which spectral characteristics (energy, phase) of each window are stored in memory to be utilised later for reconstructing free surface elevation and velocities. Since the DFT algorithm requires the signal to be periodic, each window is filtered at the leading and tailing part using a cosine taper window (Harris 1978) according to Equation 7:

$F(t)=\left\{\begin{array}{cc}0.5\left[1+\cos \pi\left(\frac{t-t_{1}}{c}-1\right)\right] & t \leq t_{1}+T_{\text {filt }} \\ 1 & t \geq t_{1}+T_{\text {filt }} \text { and } t \leq t_{2}-T_{\text {filt }} \\ 0.5\left[1+\cos \pi\left(\frac{t_{2}-t}{c}-1\right)\right] & t \geq t_{2}-T_{\text {filt }}\end{array} \quad t \in\left[t_{1}, t_{2}\right]\right.$

where $t_{1}, t_{2}$ are the start and end times of the window interval and $T_{\text {filt }}$ is the duration of the filtering length. The windows need to overlap with each other, to avoid loss of energy, as the filtered leading and tailing part are filtered. A handover point is set in the overlap area for switching to the next window and this is defined following requirement for coupling with the relaxation zone (see Section 2.4). A graphical representation of the process is shown in Figure 1. 


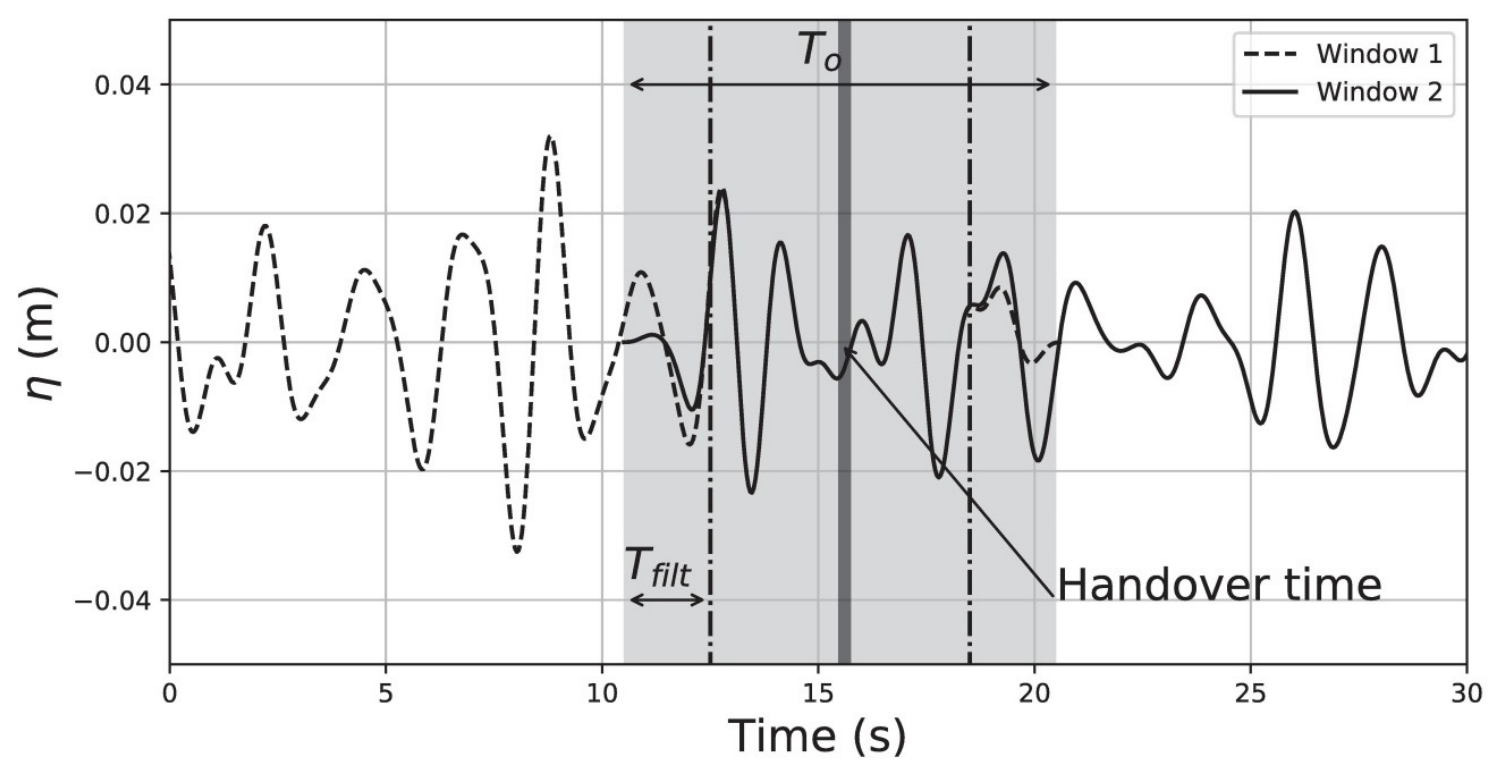

Figure 1: An example of spectral windowing method applied to reconstruct a random signal. Time series represented with dashed and solid lines red colour correspond to consecutive windows. The overlap area is highlighted with light grey, filtered areas with dark grey and the overlap point with a vertical dashed line.

Due to the shorter span interval of each window, less frequency components are necessary to ensure high fidelity reconstruction of the time series. The reconstructed free surface elevation and wave velocities are calculated by equations $8-10$, respectively.

$\eta(\vec{x}, t)=\sum_{i=1}^{N} a_{i}^{N w(t)} \cos \left(\vec{k}_{i} \cdot \vec{x}-\omega_{i} t+\phi_{i}^{N w(t)}\right)$

$U_{h}(\vec{x}, z, t)=\sum_{i=1}^{N} u_{h(i)}^{N w(t)} \cos \left(\vec{k}_{i} \cdot \vec{x}-\vec{\omega}_{i} t+\phi_{i}^{N w(t)}\right) \cosh \left(k_{i}(d+z)\right)$

$U_{v}(\vec{x}, z, t)=\sum_{i=1}^{N} u_{v(i)}^{N w(t)} \sin \left(\vec{k}_{i} \cdot \vec{x}-\vec{\omega}_{i} t+\phi_{i}^{N w(t)}\right) \sinh \left(k_{i}(d+z)\right)$

where $a_{i}^{N w}, \phi_{i}^{N w(t)}, u_{h(i)}^{N w(t)}$ and $u_{v(i)}^{N w(t)}$ are the wave amplitude, phase, horizontal and vertical velocity amplitude, respectively, for the $i$-th wave component and the $N w(t)$-th window. The index $N w(t)$ depends on the time variable.

To further enhance computational speed the optimisations discussed in Dimakopoulos et al 2016 were applied. Furthermore, the hyperbolic functions in the wave velocity profiles are also approximated using a fast $10^{\text {th }}$ order Taylor approximation. The approximation for the hyperbolic functions is applied up to a relative depth of one wavelength of component. Below this depth, velocities the wave dynamics are set to zero, as wave kinematics are expected to be relatively insignificant. 


\subsection{Relaxation zone method}

Wave generation and absorption is achieved using the relaxation zone method. The role of the relaxation zone is to gradually diffuse the boundary conditions within transitional areas using suitably selected weighting functions. This has been proven to absorb waves propagating outside of the numerical domain, both at the inlet (active absorption) and the outlet (passive absorption), so long as an appropriate length is used for the transitional zones (typically one or two wavelengths). In Proteus, only the velocity field corresponding to boundary conditions is imposed through the relaxation zone using appropriate penalty terms in the fluid momentum equations (equation 11)

$\frac{\partial \boldsymbol{u}}{\partial t}+(\boldsymbol{u} \cdot \nabla) u=\frac{1}{\rho}(\nabla p+\nabla \tau)+\boldsymbol{g}+\alpha\left(\boldsymbol{u}-\boldsymbol{u}_{\boldsymbol{t}}\right)$

where $t$ is the time variable, $\boldsymbol{u}$ and $\boldsymbol{u}_{\boldsymbol{T}}$ are the field and boundary velocity vectors, respectively, $p$ the pressure, $\tau$ the shear stress, $\boldsymbol{g}$ the gravity vector, $\rho$ the density, $\alpha$ is the penalty term coefficient and $\nabla=$ $\left(\frac{\partial}{\partial x}, \frac{\partial}{\partial y}, \frac{\partial}{\partial z}\right)$, with $x, y, z$ being the spatial coordinates. Note that penalty terms are essentially source terms that create a tendency to the solution to converge to a known value. Using larger penalty coefficients increases this tendency.

In order to determine the impact of the penalty term to the momentum equations, a dimensional analysis is performed according to the following assumptions for dimensional scaling:

- $t^{\prime}=t \cdot T$, where $T$ is the wave period

- $\left(x^{\prime}, y^{\prime}, z^{\prime}\right)=c T \cdot(x, y, z)$ where $c$ is the wave celerity

- $\left(\boldsymbol{u}^{\prime}, \boldsymbol{u}_{t}^{\prime}\right)=U\left(\boldsymbol{u}, \boldsymbol{u}_{\boldsymbol{t}}\right)$ where $U$ is a typical magnitude of the particle velocity

- $\left(\tau^{\prime}, p^{\prime}{ }_{d}\right) \sim \rho U^{2}\left(\tau, p_{d}\right)$ where $p_{d}=p-\rho(g \cdot \boldsymbol{x})$, with $\boldsymbol{x}=(x, y, z)$

ㅁ $a^{\prime}=a \cdot T$

Note that the dimensional analysis is applied in the water phase, where wave propagation takes place. Applying these scaling consideration results in equation 12 , which is a non-dimensional form of equation 11 :

$\frac{\partial \boldsymbol{u}^{\prime}}{\partial t}+\frac{U}{c}\left(\boldsymbol{u}^{\prime} \cdot \nabla\right) u^{\prime}=\frac{U}{c}\left(\nabla p_{d}^{\prime}+\nabla \tau^{\prime}\right)+a^{\prime}\left(\boldsymbol{u}^{\prime}-\boldsymbol{u}_{\boldsymbol{t}}^{\prime}\right)$

According to the dimensional analysis all variables in Equation 12 are $O(1)$ and therefore the relative importance of each equation term depends on the coefficients used for the dimensional scaling. Note that for nonbreaking waves, the particle velocity is always smaller than the celerity, so $0<\frac{U}{c}<1$. Since $\frac{U}{c}$ is also generally $O(1)$ or less, the relative importance of the penalty term is controlled by $a^{\prime}$. When $a^{\prime} \simeq 0$, then the penalty term has no influence over the momentum equation, hence the velocity is calculated by the numerical solution. When $a^{\prime} \gg 1$, the penalty term is dominant, and therefore the solution for the velocity will 
converge to $\boldsymbol{u}^{\prime}=\boldsymbol{u}_{\boldsymbol{t}}{ }_{\boldsymbol{t}}$. Note that $\boldsymbol{u}_{\boldsymbol{t}}{ }_{\text {. }}$ can be either calculated from wave theories, thus acting as active wave absorption, or set to zero for passively absorbing outgoing waves at the outlet.

The non-dimensional term $a^{\prime}$ is equivalent to the dissipation term used in Nwogu and Demirbilek (2001) for the BOUSS2D model, where in essence they propose that $a^{\prime}=30$ is sufficient for imposing $\boldsymbol{u}^{\prime}=\boldsymbol{u}^{\prime}{ }_{t}$ at the computational nodes next to the outlet boundary. At the interface of the relaxation zone and the numerical domain the solution is calculated by the momentum equation without the penalty terms, therefore $a^{\prime}=0$. The transition from $a^{\prime}=0$ to $a^{\prime} \gg 1$ in the relaxation zone is achieved by using a suitable function. By rewriting equation 11 to take into account the analysis above, it is shown that:

$\frac{\partial \boldsymbol{u}}{\partial t}+(\boldsymbol{u} \cdot \nabla) u=\frac{1}{\rho}(\nabla p+\nabla \tau)+\boldsymbol{g}+\frac{\sigma a^{\prime}}{T}\left(\boldsymbol{u}-\boldsymbol{u}_{\boldsymbol{t}}\right)$

where $\sigma$ is a relaxation function varying from 0 to 1 along the length of the relaxation zone. There are various forms of this function proposed in the literature, herein we will use the one proposed in Jacobsen et al. (2012) as it has been shown to efficiently absorb waves by both Jacobsen et al (2012) and Dimakopoulos et al. (2016).

Equation 10 is numerically implemented using the existing capability of Proteus for modelling flows in porous regions (Dimakopoulos et al 2017a), as the penalty term could be alternatively be considered as a Darcytype term for porous losses. The relaxation zones are defined as separate porous domains, where the porosity is assumed to be unity and the porous dissipation terms are set according to the penalty terms in Equation 10 . Note that $\boldsymbol{u}_{\boldsymbol{t}}$ in the air phase is set by default to zero.

\subsection{Coupling window reconstruction with relaxation zone}

According to the window reconstruction method (Section 2.2), as the simulation advances in time, free surface elevation and velocities are calculated as shown in Equation 6 and Equation 7, respectively. The phase and amplitude of linear wave components depend on the active time window. The active window is tracked by comparing the simulation time against the original free surface elevation time series. As the original time series is provided for a single point in space, the spatial dependency term $\vec{k} \cdot \vec{x}$ is constant for all linear components and can therefore be eliminated during the window processing stage, by conveniently setting the location of the series to coincide with the wave generation boundary.

However, when using the relaxation zone method, the influence of the spatial dependency term must be taken into account, as the analytical solution is calculated for every mesh cell inside the relaxation zone. The spatial variability is then introduced as a phase lag in the calculation of each linear component for both free surface and velocity, according to the following equations:

$$
\begin{aligned}
& \eta(\vec{x}, t)=a_{i}^{N w(t)} \cos \left(-\omega_{i}\left(t-t_{L(i)}\right)+\phi_{i}^{N w(t)}\right) \\
& U_{h}(\vec{x}, z, t)=u_{h(i)}^{N w(t)} \cos \left(-\omega_{i}\left(t-t_{L(i)}\right)+\phi_{i}^{N w(t)}\right) \cosh \left(k_{i}(d+z)\right)
\end{aligned}
$$


$U_{v}(\vec{x}, z, t)=u_{v(i)}^{N w(t)} \sin \left(-\omega_{i}\left(t-t_{L(i)}\right)+\phi_{i}^{N w(t)}\right) \sinh \left(k_{i}(d+z)\right)$

where $t_{L(i)}=\vec{k}_{i} \cdot \vec{x} / \omega_{i}$ is the temporal lag due to the change of spatial coordinates. The temporal lag $t_{L(i)}$ depends on the frequency component and the spatial coordinates and increases moving further away from the generation boundary and / or using higher wave frequency components to reconstruct the wave field.

A graphical representation of the effect of the temporal lag term in the window method is shown in Figure 2. In the top panel, the black solid-dot line represents the temporal lag between the wave generation boundary for a given location in the relaxation zone for the i-th frequency component. As the simulation time crosses the handover point between two overlapping windows (see bottom panel), the wave field at the generation boundary is calculated from the spectral properties of the subsequent window. Due to the temporal lag, the wave field in a downstream part of the relaxation zone may still need to be computed using the spectral properties of the previous window. This may eventually cause inconsistencies during the reconstruction of the wave field in the relaxation zone.

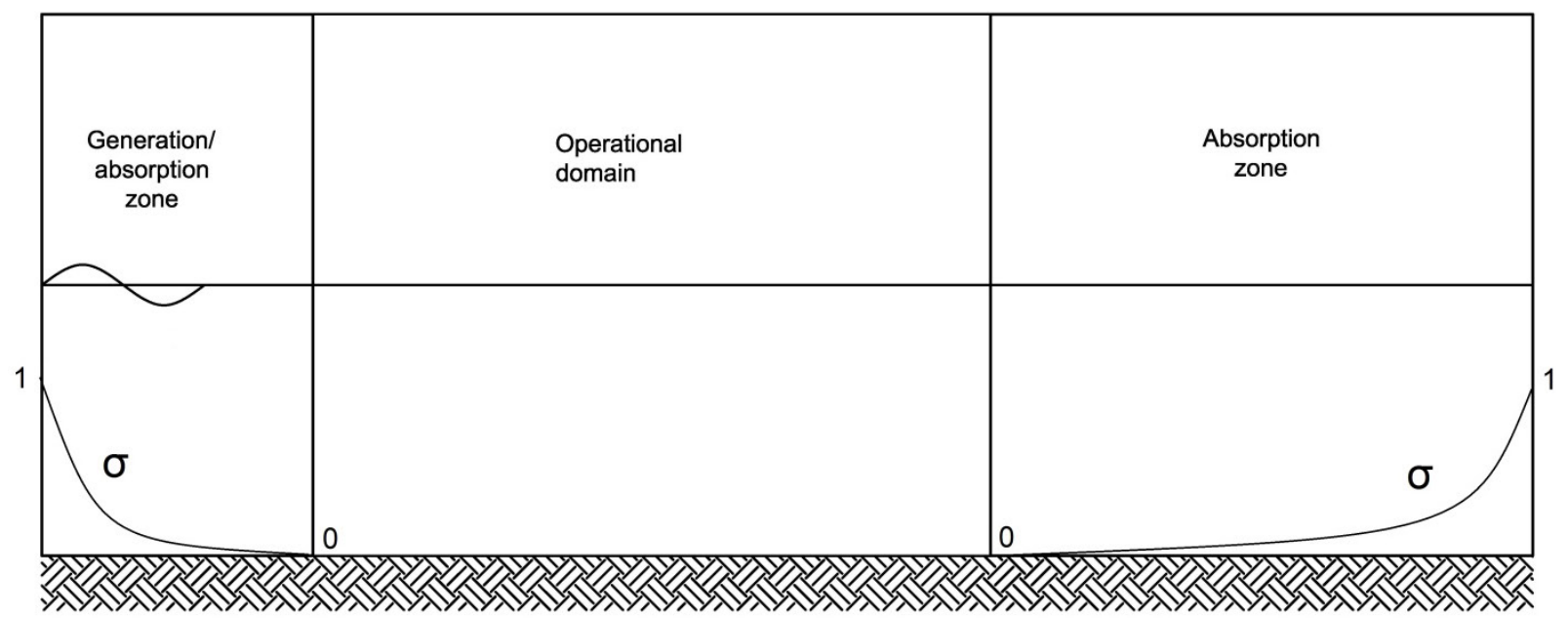

Figure 2: Effect of the temporal lag in window processing. Top: numerical wave tank with boundary $(t)$ and internal $\left(t-t_{L}\right)$ points in the generation / absorption zones. Bottom: Temporal lag between $t$ and $t-t_{L}$ in the context of the window method for wave generation

In order to eliminate these inconsistencies, either of the two following conditions should be met for the maximum value the temporal lag $\left(t_{L(\max )}\right)$ in the relaxation zone:

- $t-t_{L(\max )}$ and $t$ belong to the same window; or

- $t-t_{L(\max )}$ and $t$ belong to adjacent windows and are both found in the common overlap area, excluding filtered areas

The second requirement can be only met if the following restriction is valid

$t_{L(\max )} \leq \frac{T_{o}}{2}-T_{\text {filt }}$ 
where $T_{o}$ and $T_{\text {filt }}$ are the duration of the overlap and filtering areas, respectively, in Figure 2.

The recommended values of $t_{\mathrm{L}(\max )}, \mathrm{T}_{\text {filt }}, \mathrm{T}_{\mathrm{o}}$ depend on the configuration of the numerical tank and the number of frequency components. It was found that for a typical configuration (length of generation zone is 0.5 to 1 wavelength and up to 64 frequency components), setting $\mathrm{T}_{\text {filt }}$ and $\mathrm{T}_{\mathrm{o}}$ to 0.7 and 0.1 of the total window duration, respectively, meets equation 17 and ensures a smooth evolution of the time series. These values were adopted during the benchmark and the numerical wave tank tests presented further below.

\section{Demonstration and Benchmarking of the Windowed Reconstruction Method}

The window reconstruction methodology in Section 2 is benchmarked against direct reconstruction, in terms of both speed and accuracy. Random waves of significant wave height $H_{s}=0.0125 \mathrm{~m}$ and period $T_{p}=1.94 \mathrm{~s}$ were modelled. Subsequently, the free surface elevation was constructed using band factor $b_{F}=2$ and $N=2000$ frequency components to create a statistically meaningful time series of free surface elevation corresponding to 1000 waves, according to recommendations from Section 2.1 (Equation 4). Different realisations of the benchmark were performed by using a range of parameters for windowed reconstruction. In particular we considered $N_{W}=16,32$ and 64 waves per window and $N_{F}=8,16,32$ and 64 reconstruction frequency per window. Wave phases in Equation 1 were randomly allocated once before initialising the benchmarks and then introduced to each benchmark, for consistency. Subsequently the free-surface elevation and velocities were reconstructed using i) direct reconstruction with 2000 frequency components ii) windowed reconstruction using all possible combinations of frequency components and waves per reconstruction window mentioned above (12 in total). We calculated the maximum errors of the free-surface and velocities according to the Equation (18).

$E_{(\max )}=\max \left(\frac{\left|F_{W}\left(t_{i}\right)-F_{d}\left(t_{i}\right)\right|}{F_{S}}\right)(\%)$

where $F_{w}\left(t_{i}\right)$ and $F_{d}\left(t_{i}\right)$ are variables (free-surface, velocity) reconstructed with the window method and direct method respectively and $F_{S}$ is a normalisation factor equal to the significant wave height $H_{s}$ and corresponding particle velocity $H_{S} * \frac{2 \pi}{T}$, for the free-surface elevation and velocities, respectively. Results in terms of free-surface elevation errors and speed up are shown in Figure 3 and Figure 4. 


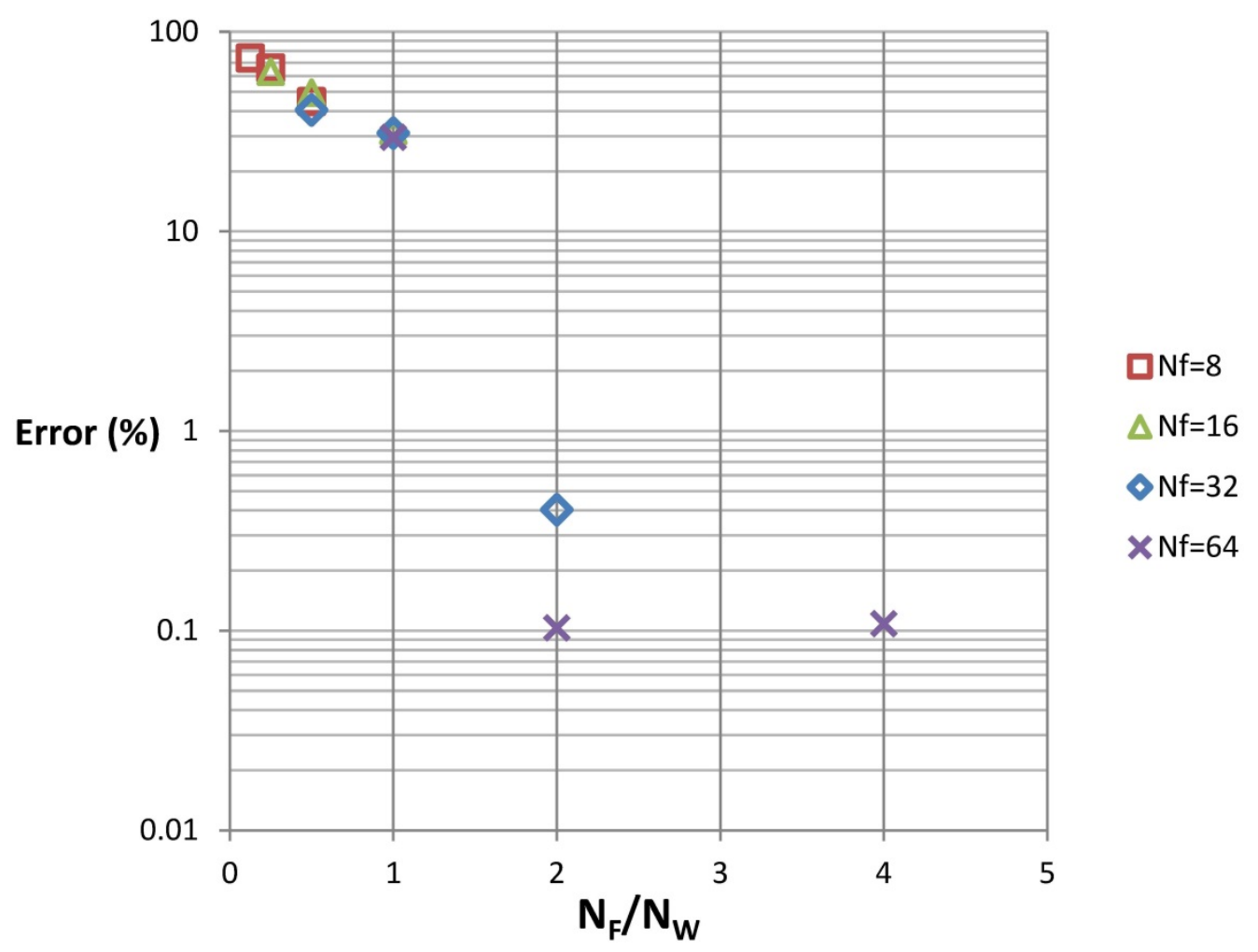

Figure 3: Maximum errors for the window reconstruction method plotted against $N_{F} / N_{W}$ for all benchmark tests.

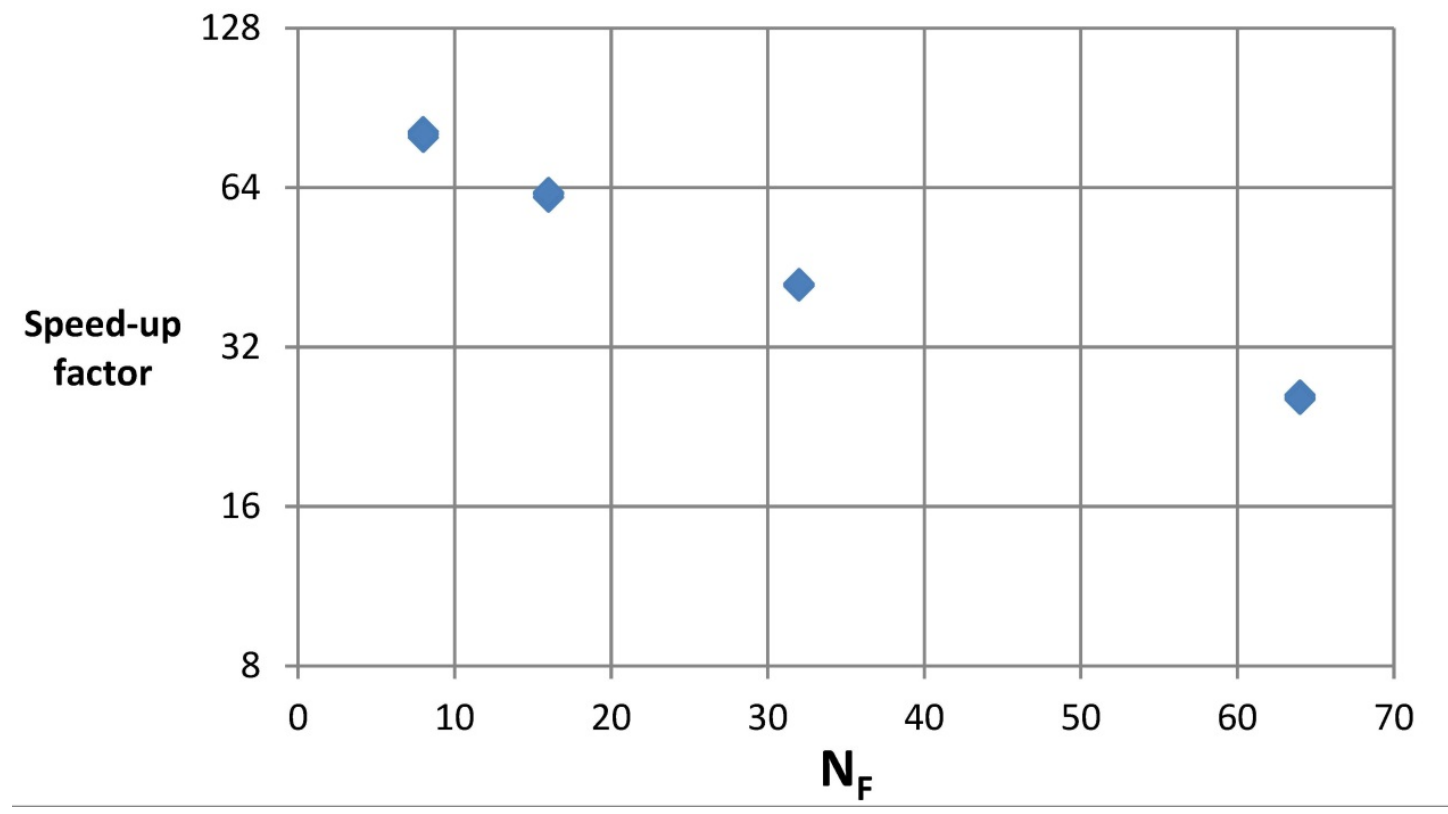

Figure 4: Speed up achieved with the window reconstruction method for the free-surface elevation calculations, plotted against the number of frequency components per window. 
In terms of errors, it is observed that for $\frac{N_{F}}{N_{W}} \geq 2$, a low error is maintained ( $\sim 0.4 \%$ for free-surface elevation and $\sim 0.2 \%$ for velocities). For lower ratios, maximum errors are unreasonable, whilst for higher ratios improvement in errors is not particularly meaningful ( $\sim .1 \%$ or less). Calculation speed-up solely depends on the number of reconstruction frequencies, and reduces with the decrease of frequencies (Figure 4). Based on the results of this benchmark, a recommended set of parameters would be $N_{F}=32$ and $N_{W}=16$. In theory, further reducing $N_{W}$ would allow $N_{F}$ and associated speed-up to be reduced. This nevertheless is not recommended, as getting $N_{W}$ close or less than $\sim 10$ may compromise the integration of the windowed reconstruction method within the relaxation zone concept which was discussed in Section 2.4. In this case $t_{L(\max )}$ will increase due to the shorter wave components whilst $T_{o}$ and $\mathrm{T}_{\text {filt }}$ will decrease due to the shorter window size, thus making difficult to accommodate the criterion set in Equation 17.

These benchmarks did not use the Taylor approximations for the trigonometric and hyperbolic functions mentioned in Section 2.1 and Dimakopoulos et al (2016), so the resulting speed-up is purely due to the different reconstruction technique. By switching on the Taylor approximations the speed-up is further improved by a factor of $\sim 2$. The maximum errors of the wave height are not significantly affected, being $0.3 \%$, whilst the maximum velocity error at the free-surface is increased $1.1 \%$.

Note that this improvements only refer to the speed-up in the calculations performed in the relaxation zone method. The overall speed-up of the simulation is less than the one reported in this section and it will be reported in the next section, along with the random wave results.

The window reconstruction method was therefore successfully applied for a linear reconstruction of components for random waves which is the predominant method used in both CFD and physical modelling studies. There is an ongoing discussion in the research and engineering community for the use of $2^{\text {nd }}$ order nonlinear correction for wave generation (Orszaghova et al. 2014), which would require the generation of wave components outside of the primary frequency band (see Tucker 1995, Schaffer 1996 and Dalzell 1999). The number of operations for performing the calculation of these components would be $\mathrm{O}\left(\mathrm{N}^{2}\right)$ rather than $\mathrm{O}(\mathrm{N})$, thus severely aggravating computational cost. In this case, the window method could be employed to significantly reduce computational cost. A potential way forward would be to assume separate representative frequency bands and process each frequency band with the windowed reconstruction method, using different window properties, tailored to the characteristic frequencies of each band. Inclusion of nonlinear corrections will be pursued as future work.

\section{Numerical tests}

Random waves were generated by using the window reconstruction method described in Section 2. A numerical wave tank was used with operational length of $15 \mathrm{~m}$ and total height of $2 \mathrm{~m}$. Random waves were generated with peak period $T_{p}=1.94 \mathrm{~s}$ (mean period $T_{m} \simeq 1.76 \mathrm{~s}$ ), significant wave height of $0.15 \mathrm{~m}$ at water depth $d=1 \mathrm{~m}$. The configuration of the wave flume is presented in Figure 2 (top panel). The generation and absorption zones are $5 \mathrm{~m}$ and $10 \mathrm{~m}$ long corresponding to one and two wavelengths, respectively, calculated using the peak period. The mesh is uniform consisting of triangular elements with constant element size of $0.025 \mathrm{~m}$. The time step was restricted using a maximum Courant number of 0.5 .

Initially, simulations were performed to compare the overall computational cost by using both the direct and the windowed reconstruction method. The aforementioned numerical tank was used, assuming a coarser mesh $(0.05 \mathrm{~s})$ and a short simulation duration. The simulations were run both in serial and parallel execution and results of the net time per $1 \mathrm{~s}$ of simulation are shown in Table 1. These values do not include any 
initialisation time, so the calculation was performed only for the time advancing loop of the algorithm. In any case, initialisation costs are similar, as these are mostly controlled by the extent of the mesh refinement and are typically a very small proportion of the overall simulation time.

Table 1: Computational cost in seconds per second of simulation for direct and window reconstruction methods

\begin{tabular}{|l|c|c|}
\hline Execution method & $\begin{array}{c}\text { Direct method } \\
\text { (s/s) }\end{array}$ & $\begin{array}{c}\text { Window method } \\
\text { (s/s) }\end{array}$ \\
\hline Serial & 618 & 390 \\
\hline Parallel (12 processors) & 202 & 66 \\
\hline
\end{tabular}

It is observed that the window reconstruction method yields an improvement of $40 \%$ and $70 \%$ to the total simulation time for serial and parallel execution respectively. The improvement is much better for parallel simulation. This is expected, as the mesh cells are equally distributed to each processor by the current CFD model and the processors containing the relaxation zone cells will face a larger computational burden, particularly for the direct reconstruction case. This computational burden is moderated by employing the window reconstruction method. This could be further improved by tackling load imbalance by using algorithms that can dynamically allocate mesh cells to each processor so that the workload is equally distributed, but such improvements are not discussed here in.

The overall computational cost improvements depend on the relative size of the relaxation zone with respect to the total domain size. In our case, the relaxation zone takes up $17 \%$ of the overall domain, which is representative of a typical configuration for simulating engineering applications. Results suggest significant improvement in computational costs, particularly for parallel runs, thus demonstrating the advantages of the use of the window reconstruction method.

Subsequently, we extended the parallel runs up to $10 \mathrm{~s}$, in order to perform a comparison between the direct and the window reconstruction method. We recorded the free-surface evolution at $\mathrm{x}=7.5 \mathrm{~m}$ and results are presented in Figure 5 and we can see very good agreement between two methods. Slight differences observed can be due to the differences between the two methods, but also because the position of the triangular mesh elements may be slightly different between the two cases, due to the way that the triangular mesh generation is performed. 


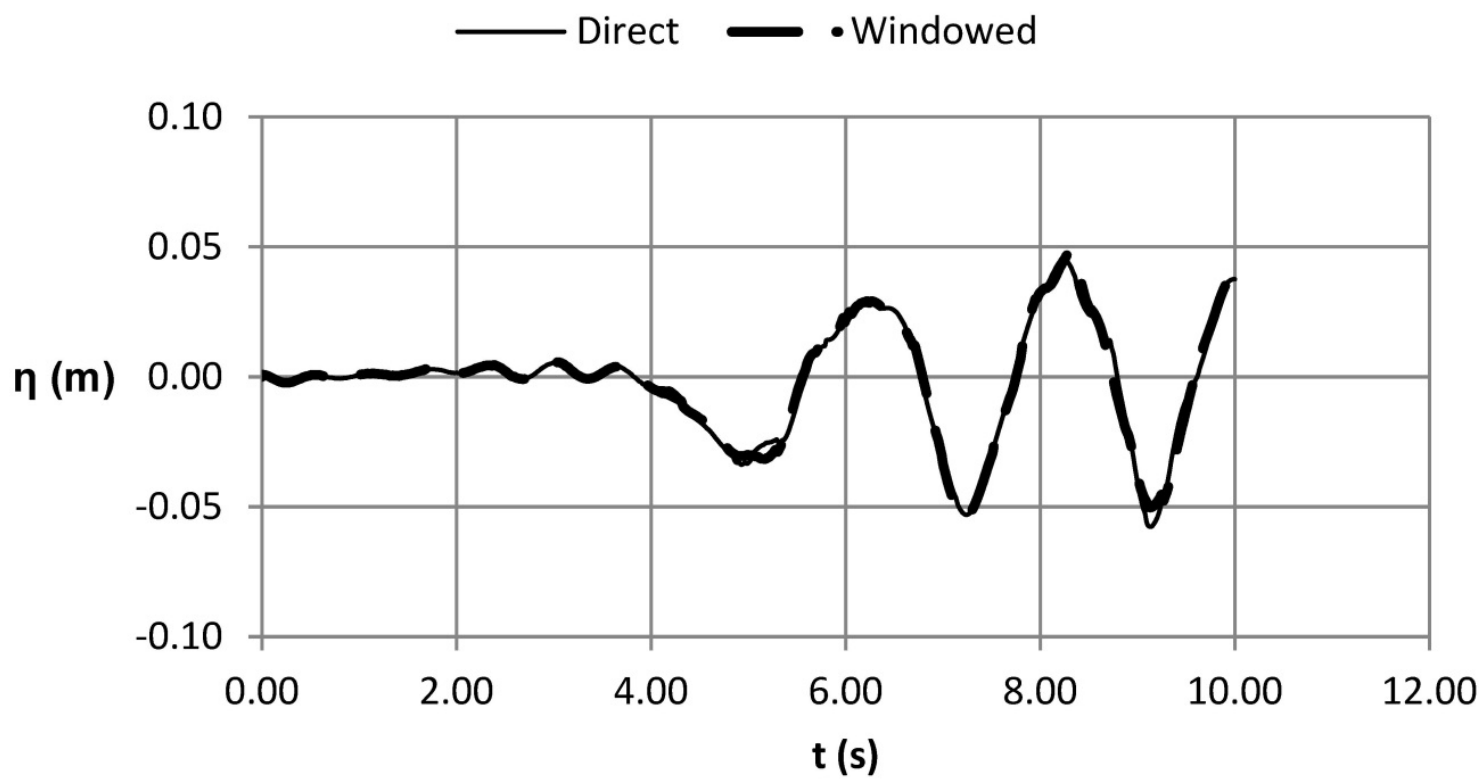

Figure 5: Free-surface elevation recorded in the numerical wave tank $(x=7.5)$ for the direct and the

A more meaningful assessment of the performance of the window reconstruction method can be achieved by performing a long simulation and calculating wave spectra and statistics. The simulation was run for $800 \mathrm{~s}$ ( 470 waves) and free-surface elevation was recorded in the flume. Figure 6 shows comparison of the freesurface elevation between Proteus and the analytical reconstruction calculations using Equation 6 . The free surface elevation is shown at four locations i) end of the generation zone ( $x=0)$, ii) $x=5 \mathrm{~m}$ ii) $x=7.5 \mathrm{~m}$ and iii) $x=10 \mathrm{~m}$. The RMS errors for the particular time window are $7.2 \% 9.7 \%, 10.8 \%$ and $12.2 \%$ for each location, respectively.
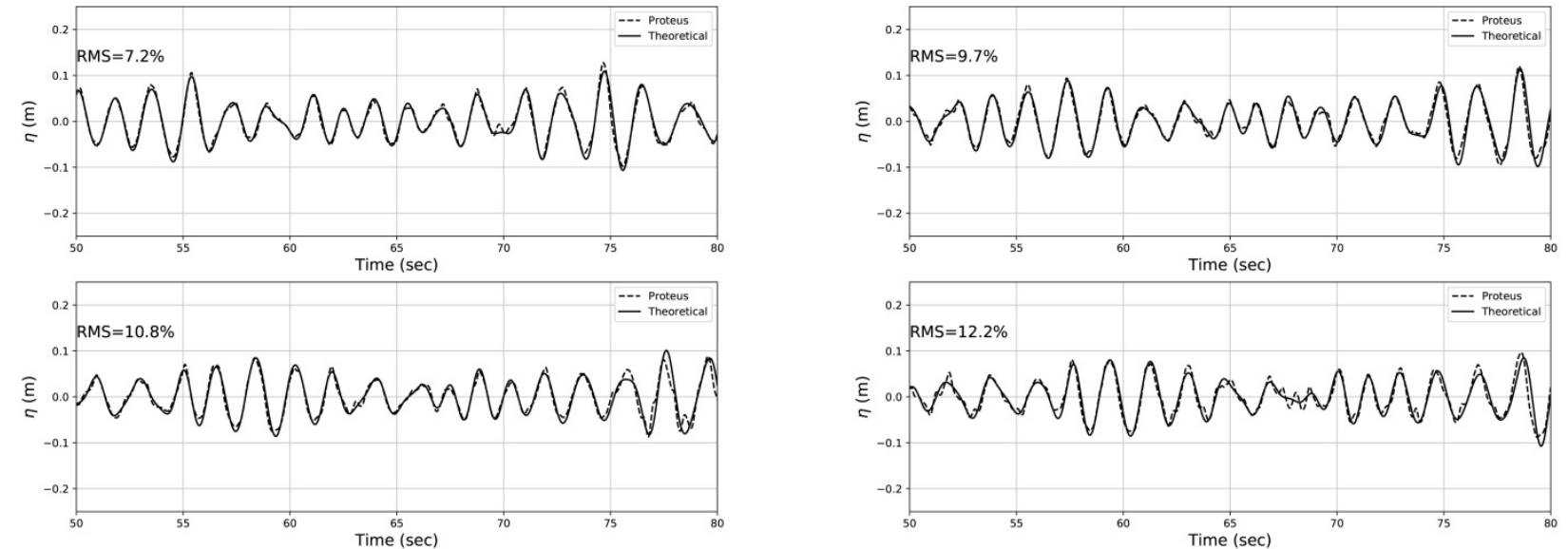

Figure 6: Random wave generation and propagation in a numerical wave flume $\left(H_{s}=0.15 \mathrm{~m}, T_{p}=1.94 \mathrm{~s}\right)$. Top left: end of the relaxation zone $(x=0)$, top right $x=5 \mathrm{~m}$, bottom left $x=7.5 \mathrm{~m}$, bottom right $x=10 \mathrm{~m}$. Dashed lines and solid lines correspond to results from Proteus and theoretical calculation using direct reconstruction method. RMS error is calculated from $10 \mathrm{~s}$ to $240 \mathrm{~s}$. 
To further investigate the capability of the model in generating random, non-repeating sequences, the wave height distribution following a wave-by-wave analysis is presented against $\sqrt{-\ln \left(1-P_{h}\right)}$, where $P_{h}$ is the probability of exceedance of each wave height in the top panel of Figure 7 . The distribution is plotted at the same four locations as in Figure 5, and the statistical analysis is performed for the last $700 \mathrm{~s}$ ( 400 waves). It is observed that the wave height statistics obey the Raleigh distribution, with the distribution being practically linear. It is also observed that wave heights are slightly reduced further away from the generation zone, which is probably due to numerical diffusion.
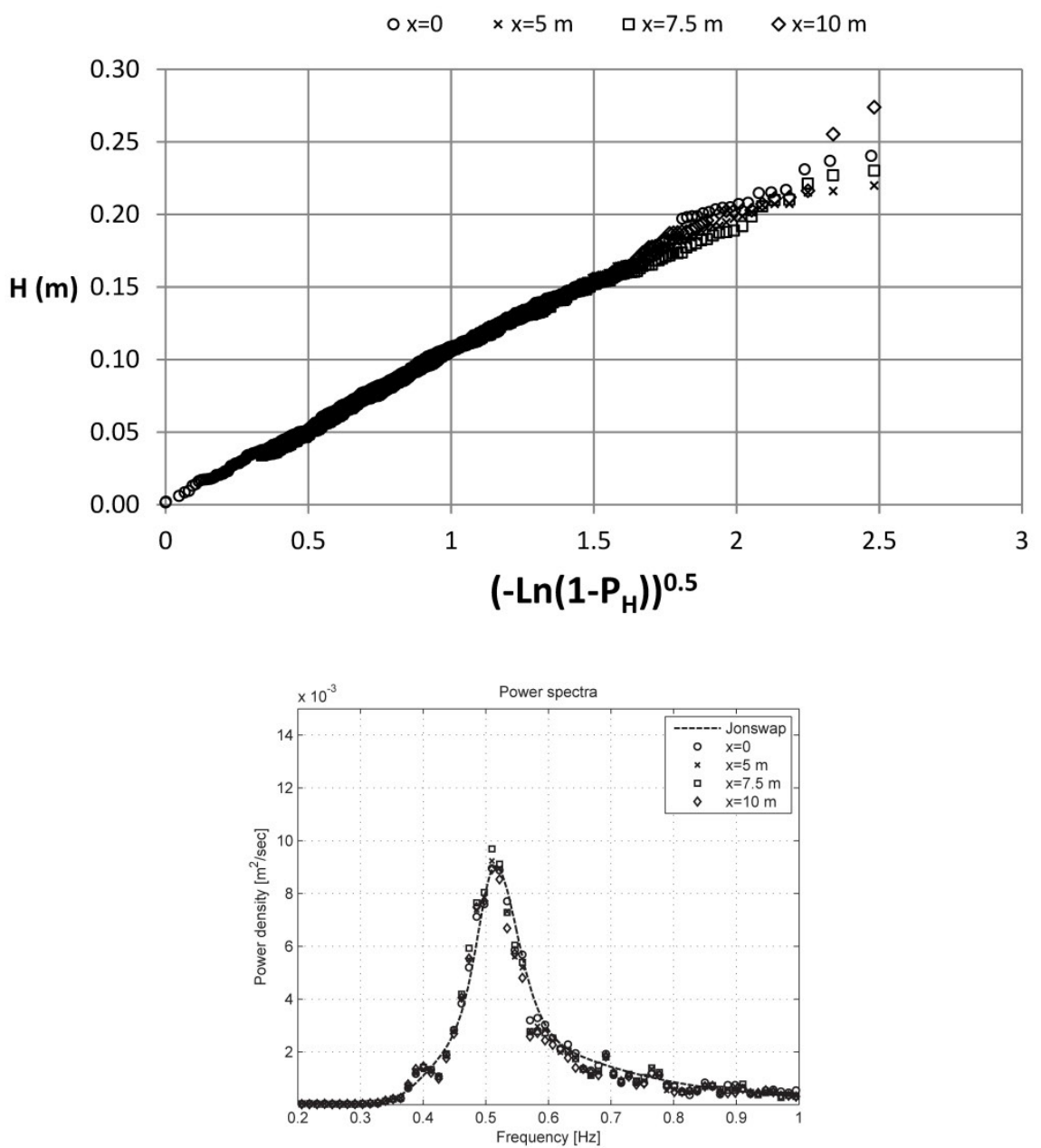

Figure 7: Top: Statistical distribution of wave heights in the random wave series $\left(H_{s}=0.15 \mathrm{~m}, T_{p}=1.94\right)$. Bottom: Spectral energy distribution, compared to the JONSWAP spectrum

The associated spectral distribution is presented in the bottom panel of Figure 7. The agreement with the theoretical JONSWAP spectrum is satisfactory, with the discrepancies owing to the relatively limited number of waves simulated. The spectral energy reduction is also evident further away from the generation, probably due to numerical diffusion. Following a 4-probe spectral analysis, the average reflection along the operating domain of the flume coefficient was found to be $7 \%$. The spectrum shows that the peak period and overall shape of the spectrum are captured very well, with the peak period predicted at $1.96 \mathrm{~s}$ ( $1 \%$ error). Some deviations are observed in the longer and short frequency areas. These are expected as most of the waves 
will be in the nonlinear regime and therefore will be subject to nonlinear interactions that will alter the shape of the original spectrum.

After applying a zero crossing analysis, the conservation of significant wave height $H_{S}$ (average height of the highest third of the wave sequence) are presented in Table 2 for each location. Overall, the errors observed in Table 2 are less that the RMS errors in Figure 6 and this is probably because the RMS errors also include dispersion errors concerning the accuracy of the prediction of the wave celerity. Indeed, in Figure 6 there is a noticeable shift of the time series in the internal locations $(x \geq 10 \mathrm{~m})$. In the context of modelling wave structure interaction processes, dispersion errors are not that significant, as long as there is a satisfactory conservation of the wave height and the peak period (shown in Figure 7). Wave period is relatively well predicted whilst wave height appears to be reduced by $2 \%$ on average.

Errors in wave height are generally caused by numerical diffusion errors, as these contribute to energy loss (e.g. see Dimakopoulos 2016). These tend to increase further away from the generation zone, and this tendency is apparent in our case, subject to some fluctuations due to the randomness of the signal.

Table 2: Peak period and zero crossing significant wave height and ( $T \_p=1.94 \mathrm{~s}$ and $\mathrm{H} \_\mathrm{s}=0.15 \mathrm{~m}$ )

\begin{tabular}{|c|c|c|c|c|c|c|}
\hline Location & $\begin{array}{c}\qquad T_{p}(\mathrm{~s}) \\
\text { (theoretical) }\end{array}$ & $\begin{array}{c}T_{p}(\mathrm{~s}) \\
\text { (Proteus) }\end{array}$ & $\begin{array}{c}\text { Error } \\
(\%)\end{array}$ & $\begin{array}{c}\qquad H_{3}(\mathrm{~s}) \\
\text { (theoretical) }\end{array}$ & $\begin{array}{c}\mathrm{H}_{3}(\mathrm{~s}) \\
\text { (Proteus) }\end{array}$ & Error (\%) \\
\hline$x=0$ & \multirow{5}{*}{1.94} & \multirow{5}{*}{1.96} & \multirow{5}{*}{1.0} & 0.148 & 0.146 & -1.4 \\
\hline$x=5 \mathrm{~m}$ & & & & 0.148 & 0.145 & -2.0 \\
\hline$x=7.5 \mathrm{~m}$ & & & & 0.148 & 0.142 & -4.1 \\
\hline$x=10 \mathrm{~m}$ & & & & 0.148 & 0.147 & -0.7 \\
\hline Mean & & & & 0.1480 & 0.1450 & -2.0 \\
\hline
\end{tabular}

\section{Discussion and recommendations}

In this paper, a methodology for generating long non-repeating sequences of random waves and associated field variables (free-surface, velocity) is presented, suitable for use in computational models. The methodology utilises spectral window processing and it is proven that this allows the reconstruction of wave sequences comprising 1000 wave events by using $O(10)$ frequencies rather than $O(1000)$, which yields significant reductions in calculation times. By additionally applying optimisations techniques for reducing the time involved in calculating trigonometric and hyperbolic functions (e.g. see Dimakopoulos et al. 2016), the calculation times are further reduced, thus making the methodology approximately two orders of magnitude faster than a direct reconstruction method using standard $\mathrm{C}++$ trigonometric and hyperbolic functions. In any case, the approximation errors remain low, with the maximum error against the original reconstruction technique being $1.1 \%$ or less. 
This methodology was developed within the frame of an open-source CFD toolkit, Proteus, based on the finite element method. The toolkit is able to model two-phase incompressible free-surface flow using a coupled VOF-level set scheme. The methodology for fast wave generation presented herein is most suitable for relaxation zone methods, as in this case the calculation of the wave field is performed in multiple cells in the domain. A relaxation zone method is therefore employed in the current model. Numerical tests were performed simulating long random wave sequences in order to investigate the overall benefit of in terms of the simulation time and the coherency of the wave field in the wave flume. In general, results are satisfactory, as the target wave field match reasonably well the theoretical one imposed as a boundary condition and the spectral and statistical characteristics were reasonably well maintained in particular for the most energetic part of the wave series. In addition, the use of the window reconstruction method allows for a reduction from $40 \%$ to $70 \%$ to the total simulation time for the case study tested herein, which is representative of a typical numerical wave tank used for engineering applications.

\section{Acknowledgements}

The authors gratefully acknowledge the financial support from ERDC and HR Wallingford through the collaboration agreement (Contract No. W911NF-15-2-0110). The first author would like to thank Ms Marine Guyot, intern at HR Wallingford for her assistance in developing early ideas of the window reconstruction method. The second author would like to acknowledge support from the IDCORE program from the Energy Technologies Institute and the Research Councils Energy Programme (grant number EP/J500847/). Permission was granted by the Chief of Engineers to publish this information.

\section{References}

Chen Q, Kelly D.M. , Dimakopoulos A.S., Zang J (2016). Validation of the PICIN solver for 2D coastal flows, Coastal Engineering, 112, pp. 87-98.

Cuomo G, Hancock, D. and Richardson S. and Longo D. and Rigden T. and Dimakopoulos A. (2013) Rehabilitation of Hartlepool town wall. In proceedings of Coasts, Marine Structures and Breakwaters.

Dalzell F. J. (1999). "A note on finite depth second-order wave-wave interactions", Applied Ocean Research, 21, 105-111.

Dean G. R., Dalrymple A. R, (1994). "Water Wave Mechanics for Engineers and Scientists", Advanced Series on Ocean Engineering, Volume 2, World Scientific x.

Dimakopoulos A.S., Cozzutto G., de Lataillade T., Kees C.E., Cuomo G. (2017) Numerical modelling of caisson breakwaters under sliding and overturning motion using the Proteus toolkit, accepted in ICE Breakwaters, Liverpool

Dimakopoulos A.S., Cozzutto G., de Lataillade T., Kees C.E., Alderson J. and Richardson S. (2017) Modelling fully coupled fluid structure interaction using the computational toolkit Proteus, accepted in Coasts \& Ports 2017 Conference - Cairns

Dimakopoulos A., Cuomo G and Chandler I. (2016). Optimized Generation and Absorption for ThreeDimensional Numerical Wave and Current Facilities. Journal of Waterway, Port, Coastal and Ocean Engineering, 06016001. 
Jacobsen N.G., Fuhrman D.R. and Fredsoe J. (2012). "A wave generation toolbox for the open-source CFD library: OpenFOAM®”. Int J of Num Meth Fluids, 70(9), pp. 1073-1088

Kees C.E., Akkerman I., Bazilevs Y., and Farthing M. W. A (2011). A Conservative Level Set Method for Variable-Order Approximations and Unstructured Meshes Journal of Computational Physics 230(12), pp. $4536-4558$

Kees C.E., Farthing M.W, Dawson C.N., (2008) Locally conservative, stabilized finite element methods for variably saturated flow, Computer Methods in Applied Mechanics and Engineering,19, pp. 4610-4625

Kees C.E., Farthing M. W., Lackey T. C., and Berger R. C. (2009) A Review of Methods for Moving Boundary Problems, Technical Report ERDC/CHL TR-09-10

Harris F.J. (1978). On the use of windows with the harmonic analysis for Fourier Transform. Proceedings of the IEEE, 66, pp. 51-83.

Higuera P., Lara J.L and Losada I.J. (2013). Realistic wave generation and active wave absorption for Navier-Stokes models. Application to OpenFOAM®. Coastal Engineering, 71(1), 102-118.

Miles M.D. and Funke E.R. (1988). Numerical comparison of wave synthesis methods, Chapter 6, Coastal Engineering Proceedings

Nwogu O. and Demirbilek Z. (2001) BOUSS-2D: A Boussinesq model, Technical report, ERDC/CHL TR-0125

Orszaghova, J, Taylor, P, Borthwick, AGL \& Raby, AC 2014, 'Importance of second-order wave generation for focused wave group run-up and overtopping' Coastal Engineering, vol. 94, pp. 63-79.

https://doi.org/10.1016/j.coastaleng.2014.08.007

Richardson S, Cuomo G. and Dimakopoulos A. and Longo D (2013). "Coastal structure optimisation using advanced numerical methods" In Proceedings of Coasts, Marine Structures and Breakwaters.

Schaffer (1996), Second-order wave makers for irregular waves, Ocean Engineering, 23 (1), pp. 47-88.

Schmitt, P \& Elsaesser, B 2015, 'A Review of Wave Makers for 3D Numerical Simulations' Paper presented at Marine 2015 VI International Conference on Computational Methods in Marine Engineering, Rome, Italy, 15/06/2015 - 17/06/2015, .

Tucker J. M. (1995). Nearshore wave height during storms, Coastal Engineering, 24, pp. 111-136.

Welch, P. D. (1967), The use of Fast Fourier Transform for the estimation of power spectra: A method based on time averaging over short, modified periodograms, IEEE Transactions on Audio and Electroacoustics, 15(2), pp. 70-73. 